\title{
Research Article \\ Continuous Quasi Gyrolinear Functionals on Möbius Gyrovector Spaces
}

\author{
Keiichi Watanabe (iD \\ Department of Mathematics, Faculty of Science, Niigata University, 950-2181, Japan \\ Correspondence should be addressed to Keiichi Watanabe; wtnbk@math.sc.niigata-u.ac.jp
}

Received 10 April 2020; Accepted 16 May 2020; Published 18 June 2020

Academic Editor: Calogero Vetro

Copyright ( 2020 Keiichi Watanabe. This is an open access article distributed under the Creative Commons Attribution License, which permits unrestricted use, distribution, and reproduction in any medium, provided the original work is properly cited.

We investigate a class of functionals on Möbius gyrovector spaces, which consists of a counterpart to bounded linear functionals on Hilbert spaces.

\section{Introduction}

Ungar initiated a study on gyrogroups and gyrovector spaces. Gyrovector spaces are generalized vector spaces, with which they share important analogies, just as gyrogroups are analogous to groups. The first known gyrogroup was the ball of Euclidean space $\mathbb{R}^{3}$ endowed with Einstein's velocity addition associated with the special theory of relativity (cf. [1]). Another example of a gyrogroup is the open unit disc in the complex plain endowed with the Möbius addition. Ungar extended these gyroadditions to the ball of an arbitrary real inner product space, introduced a common gyroscalar multiplication, and observed that the ball endowed with gyrooperations are gyrovector spaces (cf. [2, 3]). Although gyrooperations are generally not commutative, associative, or distributive, they are enjoying algebraic rules such as left and right gyroassociative, gyrocommutative, scalar distributive, and scalar associative laws, so there exist rich symmetrical structures which we should clarify precisely.

Abe and Hatori [4] introduced the notion of generalized gyrovector spaces (GGVs), which is a generalization of the notion of real inner product gyrovector spaces by Ungar. Hatori [5] showed several substructures of positive invertible elements of a unital $C^{*}$-algebra are actually GGVs. Abe [6] introduced the notion of normed gyrolinear spaces, which is a further generalization of the notion of GGVs. Although they are complicated objects from the viewpoint of the present article and we do not deal with them here, they will provide advanced research subjects.
In this article, we concentrate on the Möbius gyrovector spaces, because they are most fundamental among real inner product gyrovector spaces. There are notions of the Einstein gyrovector spaces and the PV gyrovector spaces by Ungar, and they are isomorphic to the Möbius gyrovector spaces, so most results on each space can be directly translated to other two spaces. In the Möbius gyrovector spaces, one can consider counterparts to various notions of Hilbert spaces such as the orthogonal decomposition and the closest point property with respect to any closed linear subspace, orthogonal expansion with respect to any orthonormal basis, and the Cauchy-Bunyakovsky-Schwarz inequality (cf. [1-3, 5, 7-14]).

We study some aspects of the Möbius gyrovector spaces from some viewpoints of basic theory of functional analysis. The celebrated Riesz-Fréchet theorem is one of the most fundamental theorems in both theory and application of functional analysis. It states that every bounded linear functional on a Hilbert space can be represented as a map taking the value of the inner product of each variable vector and a fixed vector. This fact makes duality in Hilbert spaces much closer to finite-dimensional duality, and for that reason, it is a particularly useful tool. We investigate a certain class of continuous functionals on the Möbius gyrovector spaces corresponding to linear functionals induced by the inner product and reveal analogies that it shares with the Riesz representation theorem.

The paper is organized as follows. Section 2 is the preliminaries. In Section 3, we show a triviality of continuous 
gyrolinear functionals on the Möbius gyrovector spaces. In Section 4, we investigate the relationship between the Möbius operations and the linear functionals induced by the inner product and consider a representation theorem of Riesz type. In Section 5, we present a class of continuous functionals that are induced by square summable sequences of real numbers. It can be regarded as a counterpart to continuous linear functionals on real Hilbert spaces, and we might call it quasi gyrolinear functionals.

\section{Preliminaries}

Let us briefly recall the definition of the Möbius gyrovector spaces. For precise definitions, basic results of gyrocommutative gyrogroups and gyrovector spaces, see monograph [9] or [10] by Ungar. For elementary facts on inner product spaces, for instance, one can refer to [8].

Let $\mathbb{V}=(\mathbb{V},+, \bullet)$ be a real inner product space with a binary operation + and a positive definite inner product $\bullet$. Let $\mathbb{V}_{s}$ be an open ball

$$
\mathbb{V}_{s}=\{\mathbf{a} \in \mathbb{V}:\|\mathbf{a}\|<s\}
$$

for any fixed $s>0$, where $\|\mathbf{a}\|=(\mathbf{a} \cdot \mathbf{a})^{1 / 2}$.

Definition 1 ([10], Definition 3.40, Definition 6.83). The Mö bius addition $\oplus_{M}$ and the Möbius scalar multiplication $\otimes_{M}$ are given by the equations

$$
\begin{aligned}
& \mathbf{a} \oplus_{M} \mathbf{b}=\frac{\left(1+\left(2 / s^{2}\right) \mathbf{a} \cdot \mathbf{b}+\left(1 / s^{2}\right)\|\mathbf{b}\|^{2}\right) \mathbf{a}+\left(1-\left(1 / s^{2}\right)\|\mathbf{a}\|^{2}\right) \mathbf{b}}{1+\left(2 / s^{2}\right) \mathbf{a} \cdot \mathbf{b}+\left(1 / s^{4}\right)\|\mathbf{a}\|^{2}\|\mathbf{b}\|^{2}}, \\
& r \otimes_{M} \mathbf{a}=s \tanh \left(r \tanh ^{-1} \frac{\|\mathbf{a}\|}{s}\right) \frac{\mathbf{a}}{\|\mathbf{a}\|}(\text { if } \mathbf{a} \neq \mathbf{0}), \quad r \otimes_{M} \mathbf{0}=\mathbf{0},
\end{aligned}
$$

for any $\mathbf{a}, \mathbf{b} \in \mathbb{V}_{s}, r \in \mathbb{R}$. The addition $\oplus_{M}$ and the scalar multiplication $\otimes_{M}$ for real numbers are defined by the equations

$$
\begin{aligned}
& a \oplus_{M} b=\frac{a+b}{1+\left(1 / s^{2}\right) a b}, \\
& r \otimes_{M} a=s \tanh \left(r \tanh ^{-1} \frac{a}{s}\right),
\end{aligned}
$$

for any $a, b \in(-s, s), r \in \mathbb{R}$.

We simply denote $\oplus_{M}, \otimes_{M}$ by $\oplus_{s}, \otimes_{s}$, respectively. If several kinds of operations appear in a formula simultaneously, we always give priority by the following order: (i) ordinary scalar multiplication, (ii) gyroscalar multiplication $\otimes_{s}$, and (iii) gyroaddition $\oplus_{s}$, that is,

$$
r_{1} \otimes_{s} w_{1} \mathbf{a}_{1} \oplus_{s} r_{2} \otimes_{s} w_{2} \mathbf{a}_{2}=\left\{r_{1} \otimes_{s}\left(w_{1} \mathbf{a}_{1}\right)\right\} \oplus_{s}\left\{r_{2} \otimes_{s}\left(w_{2} \mathbf{a}_{2}\right)\right\},
$$

and the parentheses are omitted in such cases.

In the limit of large $s, s \longrightarrow \infty$, the ball $\mathbb{V}_{s}$ expands to the whole space $\mathbb{V}$. The next proposition suggests that each result in linear analysis can be recaptured from the counterpart in gyrolinear analysis.

Proposition 2 ([10], after Remark 3.41, [3], p.1054). The Mö bius addition (resp., Möbius scalar multiplication) reduces to the ordinary addition (resp., scalar multiplication) as $s \longrightarrow$ $\infty$, that is,

$$
\begin{aligned}
& \mathbf{a} \oplus_{s} \mathbf{b} \longrightarrow \mathbf{a}+\mathbf{b}(s \longrightarrow \infty), \\
& r \otimes_{s} \mathbf{a} \longrightarrow r \mathbf{a}(s \longrightarrow \infty),
\end{aligned}
$$

for any $\mathbf{a}, \mathbf{b} \in \mathbb{V}$ and $r \in \mathbb{R}$.

Definition 3 ([10], Definition 2.7, (2.1), (6.286), (6.293)). The inverse element of a with respect to $\oplus_{s}$ obviously coincides with $-\mathbf{a}$. We use the notation

$$
\mathbf{a} \Theta_{s} \mathbf{b}=\mathbf{a} \oplus_{s}(-\mathbf{b})
$$

as in group theory. Moreover, the Möbius gyrodistance function $d$ and Poincaré distance function $h$ are defined by the equations

$$
\begin{aligned}
& d(\mathbf{a}, \mathbf{b})=\left\|\mathbf{b} \odot_{s} \mathbf{a}\right\|, \\
& h(\mathbf{a}, \mathbf{b})=\tanh ^{-1} \frac{d(\mathbf{a}, \mathbf{b})}{s} .
\end{aligned}
$$

Ungar showed that $h$ satisfies the triangle inequality ([10], (6.294)).

The following identities are easy consequence of the definition. One can refer to [11], Lemma 12, Lemma 14 (i).

Lemma 4. Let $s>0$. The following formulae hold:

(i) $\left\|\mathbf{a} \oplus_{s} \mathbf{b}\right\|^{2}=\left(\|\mathbf{a}\|^{2}+2 \mathbf{a} \cdot \mathbf{b}+\|\mathbf{b}\|^{2}\right) /\left(1+\left(2 / s^{2}\right) \mathbf{a} \cdot \mathbf{b}+\right.$ $\left.\left(1 / s^{4}\right)\|\mathbf{a}\|^{2}\|\mathbf{b}\|^{2}\right)$

(ii) $(\mathbf{a} / s) \oplus_{1}(\mathbf{b} / s)=\mathbf{a} \oplus_{s} \mathbf{b} / s$

(iii) $r \otimes_{1}(\mathbf{a} / s)=r \otimes_{s} \mathbf{a} / s$

for any $\mathbf{a}, \mathbf{b} \in \mathbb{V}_{s}$ and $r \in \mathbb{R}$.

Note that the Möbius operations generally are not commutative, associative, or distributive. Furthermore, the ordinary scalar multiplication does not distribute the Möbius addition.

However, the restricted Möbius operations to the interval $(-s, s)$ together with the ordinary addition and multiplication have a familiar nature. 
Lemma 5. The following identities hold:

$$
\begin{aligned}
a \oplus_{s} b & =b \oplus_{s} a, \\
a \oplus_{s}\left(b \oplus_{s} c\right) & =\left(a \oplus_{s} b\right) \oplus_{s} c, \\
0 \oplus_{s} a & =a \oplus_{s} 0=a, \\
(-a) \oplus_{s} a & =a \oplus_{s}(-a)=0, \\
1 \otimes_{s} a & =a, \\
\left(r_{1} r_{2}\right) \otimes_{s} a & =r_{1} \otimes_{s}\left(r_{2} \otimes_{s} a\right), \\
\left(r_{1}+r_{2}\right) \otimes_{s} a & =r_{1} \otimes_{s} a \oplus_{s} r_{2} \otimes_{s} a, \\
r \otimes_{s}\left(a \oplus_{s} b\right) & =r \otimes_{s} a \oplus_{s} r \otimes_{s} b,
\end{aligned}
$$

for any $a, b, c \in(-s, s), r_{1}, r_{2}, r \in \mathbb{R}$.

It is known that the inequality $\|\mathbf{a}-\mathbf{b}\| \leq 2\left\|\mathbf{a} \ominus_{s} \mathbf{b}\right\|$ holds for any $s>0$ and any $\mathbf{a}, \mathbf{b} \in \mathbb{V}_{s}$ (for instance, see [11], Lemma 14(iii)). We have some reverse inequalities as follows.

\section{Lemma 6.}

(i) If $\mathbf{a}, \mathbf{b} \in \mathbb{V}$ and $\|\mathbf{a}\|,\|\mathbf{b}\| \leq 1 / \sqrt{2}$, then the inequality

$$
\left\|\mathbf{a} \ominus_{1} \mathbf{b}\right\| \leq 2\|\mathbf{a}-\mathbf{b}\|
$$

holds.

(ii) Let $\mathbf{a}, \mathbf{b} \in \mathbb{V}$. If we take $s>0$ sufficiently large, then the inequality

$$
\left\|\mathbf{a} \ominus_{s} \mathbf{b}\right\| \leq 2\|\mathbf{a}-\mathbf{b}\|
$$

holds.

Proof.

(i) If $\|\mathbf{a}\|,\|\mathbf{b}\| \leq 1 / \sqrt{2}$, by the classical Schwarz inequality, we have

$$
\begin{array}{r}
1-2 \mathbf{a} \cdot \mathbf{b}+\|\mathbf{a}\|^{2}\|\mathbf{b}\|^{2} \geq 1-2\|\mathbf{a}\|\|\mathbf{b}\| \\
+\|\mathbf{a}\|^{2}\|\mathbf{b}\|^{2}=(1-\|\mathbf{a}\|\|\mathbf{b}\|)^{2} \geq \frac{1}{4},
\end{array}
$$

which yields

$$
\left\|\mathbf{a} \ominus_{1} \mathbf{b}\right\|^{2}=\frac{\|\mathbf{a}\|^{2}-2 \mathbf{a} \cdot \mathbf{b}+\|\mathbf{b}\|^{2}}{1-2 \mathbf{a} \cdot \mathbf{b}+\|\mathbf{a}\|^{2}\|\mathbf{b}\|^{2}} \leq 4\|\mathbf{a}-\mathbf{b}\|^{2} .
$$

(ii) For $s>\sqrt{2} \max \{\|\mathbf{a}\|,\|\mathbf{b}\|\}$, it is easy to see

$$
\left\|\frac{\mathbf{a}}{s} \ominus_{1} \frac{\mathbf{b}}{s}\right\| \leq 2\left\|\frac{\mathbf{a}}{s}-\frac{\mathbf{b}}{s}\right\|,
$$

by (i) just established above, which implies $\left\|\mathbf{a} \ominus_{s} \mathbf{b}\right\|$ $\leq 2\|\mathbf{a}-\mathbf{b}\|$. This completes the proof.
Definition 7 ([11], Definition 32). (i) Let $\left\{\mathbf{a}_{j}\right\}_{j}$ be a sequence in $\mathbb{V}_{s}$. We say that a series

$$
\left(\left(\left(\mathbf{a}_{1} \oplus_{s} \mathbf{a}_{2}\right) \oplus_{s} \mathbf{a}_{3}\right) \oplus_{s} \cdots \oplus_{s} \mathbf{a}_{j}\right) \oplus_{s} \cdots
$$

converges if there exists an element $\mathbf{x} \in \mathbb{V}_{s}$ such that $h(\mathbf{x}$, $\left.\mathbf{x}_{j}\right) \longrightarrow 0(j \longrightarrow \infty)$, where the sequence $\left\{\mathbf{x}_{j}\right\}_{j}$ is defined recursively by $\mathbf{x}_{1}=\mathbf{a}_{1}$ and $\mathbf{x}_{j}=\mathbf{x}_{j-1} \oplus_{s} \mathbf{a}_{j}$. In this case, we say the series converges to $\mathbf{x}$ and denote

$$
\mathbf{x}=\left(\left(\left(\mathbf{a}_{1} \oplus_{s} \mathbf{a}_{2}\right) \oplus_{s} \mathbf{a}_{3}\right) \oplus_{s} \cdots \oplus_{s} \mathbf{a}_{\mathbf{j}}\right) \oplus_{s} \cdots
$$

In addition, if the sequence $\left\{\mathbf{a}_{j}\right\}_{j}$ is orthogonal, then we shortly denote

$$
\mathbf{x}=\sum_{j=1}^{\infty} \mathbf{a}_{j}
$$

Note that parentheses are not necessary in the formula above by [11], Lemma 31 .

(ii) Let $\left\{a_{j}\right\}_{j}$ be a sequence in $\mathbb{R}$ with $\left|a_{j}\right|<s$ for all $j$. We say that a series

$$
\sum_{j=1}^{\infty} a_{j} a_{j}=a_{1} \oplus_{s} a_{2} \oplus_{s} \cdots \oplus_{s} a_{j} \oplus_{s} \cdots
$$

converges if there exists $x \in \mathbb{R}$ with $|x|<s$ such that $x_{j} \longrightarrow x$, where the sequence $\left\{x_{j}\right\}_{j}$ is defined recursively by $x_{1}=a_{1}$ and $x_{j}=x_{j-1} \oplus_{s} a_{j}$. In this case, we say the series converges to $x$ and denote

$$
x=\sum_{j=1}^{\infty} a_{j} .
$$

Recently, the following Schwarz type inequality related to the Möbius operations in real inner product spaces was obtained, which is an extension of a similar type inequality obtained in a preceding paper [13]. See also [12] for a discrete Cauchy type inequality restricted to real numbers.

Theorem 8 ([14], Theorem 3.8). Let $\mathbb{V}$ be a real inner product space. For any $\mathbf{a}, \mathbf{b} \in \mathbb{V}, s>\max \{\|\mathbf{a}\|,\|\mathbf{b}\|\}$ and $\mathbf{c} \in \mathbb{V}$ with $\|\mathbf{c}\| \leq 1$, the following inequality holds:

$$
\left|\mathbf{a} \cdot \mathbf{c} \ominus_{s} \mathbf{b} \cdot \mathbf{c}\right| \leq\|\mathbf{c}\| \otimes_{s}\left\|\mathbf{a} \ominus_{s} \mathbf{b}\right\|
$$

or

$$
h(\mathbf{a} \cdot \mathbf{c}, \mathbf{b} \cdot \mathbf{c}) \leq\|\mathbf{c}\| h(\mathbf{a}, \mathbf{b})
$$


that is,

$$
\begin{aligned}
& \left|\frac{\mathbf{a} \cdot \mathbf{c}-\mathbf{b} \cdot \mathbf{c}}{1-\left(1 / s^{2}\right)(\mathbf{a} \cdot \mathbf{c})(\mathbf{b} \cdot \mathbf{c})}\right| \\
& \quad \leq s \tanh \left\{\|\mathbf{c}\| \tanh ^{-1}\left(\frac{1}{s} \sqrt{\frac{\|\mathbf{a}\|^{2}-2 \mathbf{a} \cdot \mathbf{b}+\|\mathbf{b}\|^{2}}{1-\left(2 / s^{2}\right) \mathbf{a} \cdot \mathbf{b}+\left(1 / s^{4}\right)\|\mathbf{a}\|^{2}\|\mathbf{b}\|^{2}}}\right)\right\} .
\end{aligned}
$$

The equality holds if and only if one of the following conditions is satisfied:

$$
\begin{aligned}
& \text { (i) } \mathbf{a}=\mathbf{b} \\
& \text { (ii) } \mathbf{c}=0 \\
& \text { (iii) }\|\mathbf{c}\|=1 \text { and } \mathbf{a}=\lambda \mathbf{c}, \mathbf{b}=\mu \mathbf{c} \text { for some real numbers } \lambda, \mu
\end{aligned}
$$

Remark 9. Note that $\mathbf{a} \cdot \mathbf{c} \ominus_{s} \mathbf{b} \cdot \mathbf{c} \neq\left(\mathbf{a} \ominus_{s} \mathbf{b}\right) \cdot \mathbf{c}$ in general. Moreover, the following inequality does not hold:

$$
\left|\mathbf{a} \cdot \mathbf{c} \ominus_{s} \mathbf{b} \cdot \mathbf{c}\right| \leq\left|\left(\mathbf{a} \ominus_{s} \mathbf{b}\right) \cdot \mathbf{c}\right| .
$$

Indeed, take $\mathbb{V}=\mathbb{R}^{2}, s=1$ and

$$
\begin{aligned}
& \mathbf{a}=\left(\begin{array}{c}
\frac{1}{2} \\
0
\end{array}\right), \\
& \mathbf{b}=\left(\begin{array}{c}
\frac{1}{2 \sqrt{5}} \\
\frac{1}{\sqrt{5}}
\end{array}\right), \\
& \mathbf{c}=\left(\begin{array}{l}
0 \\
1
\end{array}\right) .
\end{aligned}
$$

Then, it is immediate to check

$$
\left|\mathbf{a} \cdot \mathbf{c} \ominus_{s} \mathbf{b} \cdot \mathbf{c}\right|=\frac{1}{\sqrt{5}}>\frac{12}{17 \sqrt{5}-8}=\left|\left(\mathbf{a} \ominus_{s} \mathbf{b}\right) \cdot \mathbf{c}\right|
$$

\section{Continuous Gyrolinear Functionals}

In this section, we denote $\oplus_{1}, \otimes_{1}$ by $\oplus, \otimes$ for simplicity, respectively, and we show a triviality of continuous gyrolinear functionals on the Möbius gyrovector spaces. It is an application of the orthogonal gyroexpansion with respect to an orthonormal basis in a Hilbert space, which was established in [11]. At first, we consider an elementary system of equations with the Möbius operations restricted to real numbers.
Lemma 10. Assume that $a_{1}, a_{2} \in(-1,1), r_{1}, r_{2}, t_{1}, t_{2} \in \mathbb{R}$ and

$$
\left\{\begin{array}{l}
r_{1} \otimes a_{1} \oplus r_{2} \otimes a_{2}=t_{1} \otimes a_{1} \oplus t_{2} \otimes a_{2}, \\
r_{1} \otimes a_{1} \oplus-r_{2} \otimes a_{2}=t_{1} \otimes a_{1} \oplus-t_{2} \otimes a_{2} .
\end{array}\right.
$$

Then, at least one of the following (i) (iv) holds.
(i) $r_{1}=t_{1}$ and $r_{2}=t_{2}$
(ii) $r_{1}=t_{1}$ and $a_{2}=0$
(iii) $r_{2}=t_{2}$ and $a_{1}=0$
(iv) $a_{1}=a_{2}=0$

Proof. Note that the Möbius addition $\oplus$ is commutative and associative on the open interval $(-1,1)$. Thus, we have

$$
\begin{aligned}
r_{1} \otimes a_{1} \oplus r_{1} \otimes a_{1} & =\left(r_{1} \otimes a_{1} \oplus r_{2} \otimes a_{2}\right) \oplus\left(r_{1} \otimes a_{1} \oplus-r_{2} \otimes a_{2}\right) \\
& =\left(t_{1} \otimes a_{1} \oplus t_{2} \otimes a_{2}\right) \oplus\left(t_{1} \otimes a_{1} \oplus-t_{2} \otimes a_{2}\right) \\
& =t_{1} \otimes a_{1} \oplus t_{1} \otimes a_{1},
\end{aligned}
$$

which implies that

$$
\begin{aligned}
2 \otimes\left(r_{1} \otimes a_{1}\right) & =2 \otimes\left(t_{1} \otimes a_{1}\right), \\
\tanh \left(2 r_{1} \tanh ^{-1} a_{1}\right) & =\tanh \left(2 t_{1} \tanh ^{-1} a_{1}\right), \\
r_{1} & =t_{1}
\end{aligned}
$$

or

$$
a_{1}=0 \text {. }
$$

Moreover, together with the fact that $-\left(b_{1} \oplus b_{2}\right)=-b_{1} \oplus$ $-b_{2}$, we also obtain

$$
\begin{aligned}
r_{2} \otimes a_{2} \oplus r_{2} \otimes a_{2} & =\left(r_{1} \otimes a_{1} \oplus r_{2} \otimes a_{2}\right) \oplus\left\{-\left(r_{1} \otimes a_{1} \oplus-r_{2} \otimes a_{2}\right)\right\} \\
& =\left(t_{1} \otimes a_{1} \oplus t_{2} \otimes a_{2}\right) \oplus\left\{-\left(t_{1} \otimes a_{1} \oplus-t_{2} \otimes a_{2}\right)\right\} \\
& =t_{2} \otimes a_{2} \oplus t_{2} \otimes a_{2},
\end{aligned}
$$

and hence, $r_{2}=t_{2}$ or $a_{2}=0$. Obviously, it yields the conclusion of the lemma. This completes the proof.

Theorem 11. Let $\mathbb{V}$ be a separable real Hilbert space with $\operatorname{dim} \mathbb{V} \geq 2$. Consider the Poincaré metric $h$ on the ball $\mathbb{V}_{1}$ and the interval $(-1,1)$, respectively. If a continuous map $f: \mathbb{V}_{1} \longrightarrow(-1,1)$ satisfies

$$
f(\mathbf{x} \oplus \mathbf{y})=f(\mathbf{x}) \oplus f(\mathbf{y}),
$$

for any $\mathbf{x}, \mathbf{y} \in \mathbb{V}_{1}$, then $f(\mathbf{x})=0$ for all $\mathbf{x} \in \mathbb{V}_{1}$. 
Proof. At first, it follows from a standard argument using condition (31) and the continuity of $f$ that

$$
f(r \otimes \mathbf{x})=r \otimes f(\mathbf{x}),
$$

for any $\mathbf{x} \in \mathbb{V}_{1}, r \in \mathbb{R}$.

We may assume $\mathbb{V}$ is countably infinite dimensional. Take any complete orthonormal sequense $\left\{\mathbf{e}_{j}\right\}_{j=1}^{\infty}$ of $\mathbb{V}$. Put

$$
a_{j}=f\left(\frac{\mathbf{e}_{j}}{2}\right)(j=1,2) .
$$

We use conditions (31) and (32), by the following two ways (I) and (II)

$$
\begin{gathered}
\text { (I) } \mathbf{x}=\mathbf{y}=\frac{\mathbf{e}_{1}}{2} \oplus \frac{\mathbf{e}_{2}}{2}, \\
\text { (II) } \mathbf{x}=\mathbf{y}=\frac{\mathbf{e}_{1}}{2} \oplus\left(-\frac{\mathbf{e}_{2}}{2}\right) .
\end{gathered}
$$

In the case (I), it is easy to see that

$$
\begin{aligned}
\mathbf{x} & =\mathbf{y}=\frac{\mathbf{e}_{1}}{2} \oplus \frac{\mathbf{e}_{2}}{2}=\frac{10 \mathbf{e}_{1}+6 \mathbf{e}_{2}}{17}, \\
\mathbf{x} \cdot \mathbf{y} & =\frac{8}{17}, \\
\|\mathbf{x}\| & =\|\mathbf{y}\|=\sqrt{\frac{8}{17}} \\
1+2 \mathbf{x} \cdot \mathbf{y}+\|\mathbf{x}\|^{2}\|\mathbf{y}\|^{2} & =\frac{5^{4}}{17^{2}}, \\
\mathbf{x} \oplus \mathbf{y} & =\frac{4}{5} \mathbf{e}_{1}+\frac{12}{25} \mathbf{e}_{2} .
\end{aligned}
$$

We can express $\mathbf{x} \oplus \mathbf{y} \in \mathbb{V}_{1}$ as a gyrolinear combination of $\mathbf{e}_{1} / 2, \mathbf{e}_{2} / 2$ to obtain

$$
\left(\frac{\mathbf{e}_{1}}{2} \oplus \frac{\mathbf{e}_{2}}{2}\right) \oplus\left(\frac{\mathbf{e}_{1}}{2} \oplus \frac{\mathbf{e}_{2}}{2}\right)=r_{1} \otimes \frac{\mathbf{e}_{1}}{2} \oplus r_{2} \otimes \frac{\mathbf{e}_{2}}{2}
$$

for a unique pair of real numbers $r_{1}, r_{2}$. Put $\mathbf{x}_{1}=(4 / 5) \mathbf{e}_{1}$, $\mathbf{x}_{2}=(12 / 25) \mathbf{e}_{2}$. Applying [7], Theorem 4.2, we can rewrite

$$
\frac{4}{5} \mathbf{e}_{1}+\frac{12}{25} \mathbf{e}_{2}=\lambda_{1} \mathbf{x}_{1} \oplus \lambda_{2} \mathbf{x}_{2}
$$

where $\lambda_{j}$ are given by the formulae

$$
\begin{aligned}
\lambda_{1} & =\frac{(4 / 5)^{2}+(12 / 25)^{2}+1-\sqrt{\left((4 / 5)^{2}+(12 / 25)^{2}+1\right)^{2}-4(4 / 5)^{2}}}{2(4 / 5)^{2}} \\
& =\frac{1169-39 \sqrt{241}}{2^{3} \cdot 5^{3}} \cdot \frac{5}{4}, \\
\lambda_{2} & =\frac{(4 / 5)^{2}+(12 / 25)^{2}-1+\sqrt{\left((4 / 5)^{2}+(12 / 25)^{2}+1\right)^{2}-4(4 / 5)^{2}}}{2(12 / 25)^{2}} \\
& =\frac{-81+39 \sqrt{241}}{2^{3} \cdot 3 \cdot 5^{2}} \cdot \frac{25}{12} .
\end{aligned}
$$

Therefore, we have

$$
\begin{aligned}
\frac{4}{5} \mathbf{e}_{1}+\frac{12}{25} \mathbf{e}_{2} & =\lambda_{1} \mathbf{x}_{1} \oplus \lambda_{2} \mathbf{x}_{2} \\
& =\frac{1169-39 \sqrt{241}}{2^{3} \cdot 5^{3}} \mathbf{e}_{1} \oplus \frac{-81+39 \sqrt{241}}{2^{3} \cdot 3 \cdot 5^{2}} \mathbf{e}_{2} \\
& =r_{1} \otimes \frac{\mathbf{e}_{1}}{2} \oplus r_{2} \otimes \frac{\mathbf{e}_{2}}{2}
\end{aligned}
$$

where $r_{j}$ are given by the formulae

$$
\begin{aligned}
& r_{1}=\frac{\tanh ^{-1}\left((1169-39 \sqrt{241}) /\left(2^{3} \cdot 5^{3}\right)\right)}{\tanh ^{-1}(1 / 2)} \\
& r_{2}=\frac{\tanh ^{-1}\left((-81+39 \sqrt{241}) /\left(2^{3} \cdot 3 \cdot 5^{2}\right)\right)}{\tanh ^{-1}(1 / 2)} .
\end{aligned}
$$

On the other hand, it is easy to check

$$
\tanh \left(2 \tanh ^{-1} \frac{1}{2}\right)=2 \otimes \frac{1}{2}=\frac{2 \cdot(1 / 2)}{1+(1 / 2)^{2}}=\frac{4}{5}
$$

which implies that $r_{j} \neq 2$.

Since $f$ satisfies formulae (31) and (32), it follows from taking the value of $f$ in (36) that

$$
\begin{aligned}
2 \otimes a_{1} \oplus 2 \otimes a_{2} & =\left(a_{1} \oplus a_{2}\right) \oplus\left(a_{1} \oplus a_{2}\right) \\
& =\left\{f\left(\frac{\mathbf{e}_{1}}{2}\right) \oplus f\left(\frac{\mathbf{e}_{2}}{2}\right)\right\} \oplus\left\{f\left(\frac{\mathbf{e}_{1}}{2}\right) \oplus f\left(\frac{\mathbf{e}_{2}}{2}\right)\right\} \\
& =f\left(\left(\frac{\mathbf{e}_{1}}{2} \oplus \frac{\mathbf{e}_{2}}{2}\right) \oplus\left(\frac{\mathbf{e}_{1}}{2} \oplus \frac{\mathbf{e}_{2}}{2}\right)\right) \\
& =f\left(r_{1} \otimes \frac{\mathbf{e}_{1}}{2} \oplus r_{2} \otimes \frac{\mathbf{e}_{2}}{2}\right)=r_{1} \otimes a_{1} \oplus r_{2} \otimes a_{2}
\end{aligned}
$$

In the case (II), a similar calculation shows that

$$
\begin{gathered}
\mathbf{x}=\mathbf{y}=\frac{\mathbf{e}_{1}}{2} \oplus\left(-\frac{\mathbf{e}_{2}}{2}\right)=\frac{10 \mathbf{e}_{1}-6 \mathbf{e}_{2}}{17}, \\
\mathbf{x} \oplus \mathbf{y}=\frac{4}{5} \mathbf{e}_{1}-\frac{12}{25} \mathbf{e}_{2}, \\
\left(\frac{\mathbf{e}_{1}}{2} \oplus-\frac{\mathbf{e}_{2}}{2}\right) \oplus\left(\frac{\mathbf{e}_{1}}{2} \oplus-\frac{\mathbf{e}_{2}}{2}\right)=r_{1} \otimes \frac{\mathbf{e}_{1}}{2} \oplus-r_{2} \otimes \frac{\mathbf{e}_{2}}{2},
\end{gathered}
$$

where $r_{1}$ and $r_{2}$ are identically given by the formulae (40) and (41), respectively, and we obtain 


$$
\begin{aligned}
2 \otimes a_{1} \oplus-2 \otimes a_{2} & =\left(a_{1} \oplus-a_{2}\right) \oplus\left(a_{1} \oplus-a_{2}\right) \\
& =\left\{f\left(\frac{\mathbf{e}_{1}}{2}\right) \oplus(-1) \otimes f\left(\frac{\mathbf{e}_{2}}{2}\right)\right\} \\
& \oplus\left\{f\left(\frac{\mathbf{e}_{1}}{2}\right) \oplus(-1) \otimes f\left(\frac{\mathbf{e}_{2}}{2}\right)\right\} \\
& =f\left(\left(\frac{\mathbf{e}_{1}}{2} \oplus-\frac{\mathbf{e}_{2}}{2}\right) \oplus\left(\frac{\mathbf{e}_{1}}{2} \oplus-\frac{\mathbf{e}_{2}}{2}\right)\right) \\
& =f\left(r_{1} \otimes \frac{\mathbf{e}_{1}}{2} \oplus-r_{2} \otimes \frac{\mathbf{e}_{2}}{2}\right) \\
& =r_{1} \otimes a_{1} \oplus-r_{2} \otimes a_{2} .
\end{aligned}
$$

Therefore, if $f$ satisfies formulae (31) and (32), then we must have the system of equations

$$
\left\{\begin{array}{l}
r_{1} \otimes a_{1} \oplus r_{2} \otimes a_{2}=2 \otimes a_{1} \oplus 2 \otimes a_{2}, \\
r_{1} \otimes a_{1} \oplus-r_{2} \otimes a_{2}=2 \otimes a_{1} \oplus-2 \otimes a_{2}
\end{array}\right.
$$

From the fact that $r_{1} \neq 2, r_{2} \neq 2$ and Lemma 10, we have $a_{1}=a_{2}=0$. Since the argument above is valid for any pair of distinct members $\mathbf{e}_{j_{1}}, \mathbf{e}_{j_{2}}$, we have $f\left(\mathbf{e}_{j} / 2\right)=0$ for all $j$.

It follows from [11], Theorem 35, that an arbitrary element $\mathbf{x}$ in $V_{1}$ has an orthogonal gyroexpansion as

$$
\mathbf{x}=\sum_{j=1}^{\infty} r_{j} \otimes \frac{\mathbf{e}_{j}}{2}
$$

Thus, we obtain

$f\left(r_{1} \otimes \frac{\mathbf{e}_{1}}{2} \oplus \cdots \oplus r_{j} \otimes \frac{\mathbf{e}_{j}}{2}\right)=r_{1} \otimes f\left(\frac{\mathbf{e}_{1}}{2}\right) \oplus \cdots \oplus r_{j} \otimes f\left(\frac{\mathbf{e}_{j}}{2}\right)=0$,

for $j=1,2, \cdots$, which implies that $f(\mathbf{x})=0$ for all $\mathbf{x} \in \mathbb{V}_{1}$ by the continuity of $f$. This completes the proof.

The case of 1-dimensional real inner product space is exceptional.

Theorem 12. Let $\mathbb{V}$ be a real inner product space with dim $\mathbb{V}=1$ and let $\mathbf{e}$ be an element in $\mathbb{V}$ with $\|\mathbf{e}\|=1$.

For an arbitrary real number $c$, the formula

$$
f(t \mathbf{e})=c \otimes t
$$

for $-1<t<1$ defines a map $f: \mathbb{V}_{1} \longrightarrow(-1,1)$ which satisfies (31) and (32) for any $\mathbf{x}, \mathbf{y} \in \mathbb{V}_{1}, r \in \mathbb{R}$.

Conversely, if a map $f: \mathbb{V}_{1} \longrightarrow(-1,1)$ satisfies (31) and (32) for any $\mathbf{x}, \mathbf{y} \in \mathbb{V}_{1}, r \in \mathbb{R}$, then $c=\tanh ^{-1} f(w \mathbf{e}) / \tanh ^{-1} w$ does not depend on $0<w<1$ and $f$ is given by formula (49) for $-1<t<1$.

Proof. Let $c$ be an arbitrary real number. Suppose that the map $f: \mathbb{V}_{1} \longrightarrow(-1,1)$ is defined by the formula (49). A straightforward calculation shows that $f$ satisfies (31) and (32) for any $\mathbf{x}, \mathbf{y} \in \mathbb{V}_{1}, r \in \mathbb{R}$.

Conversely, suppose that a map $f: \mathbb{V}_{1} \longrightarrow(-1,1)$ satisfies (31) and (32) for any $\mathbf{x}, \mathbf{y} \in \mathbb{V}_{1}, r \in \mathbb{R}$. Let $0<w<1$ be a fixed number. Any element $t \mathbf{e}(-1<t<1)$ in $\mathbb{V}_{1}$ can be expressed as

$$
\begin{aligned}
t \mathbf{e} & =r \otimes w \mathbf{e}=\tanh \left(r \tanh ^{-1}\|w \mathbf{e}\|\right) \frac{w \mathbf{e}}{\|w \mathbf{e}\|} \\
& =\tanh \left(r \tanh ^{-1} w\right) \mathbf{e}
\end{aligned}
$$

by a unique real number $r=\tanh ^{-1} t / \tanh ^{-1} w$. Then, we have

$$
\begin{aligned}
f(t \mathbf{e}) & =f(r \otimes w \mathbf{e})=r \otimes f(w \mathbf{e})=\tanh \left(r \tanh ^{-1} f(w \mathbf{e})\right) \\
& =\tanh \left(\frac{\tanh ^{-1} t}{\tanh ^{-1} w} \tanh ^{-1} f(w \mathbf{e})\right) \\
& =\tanh \left(\frac{\tanh ^{-1} f(w \mathbf{e})}{\tanh ^{-1} w} \tanh ^{-1} t\right)=c \otimes t,
\end{aligned}
$$

for any $-1<t<1$. The argument above includes that the value of $c$ does not depend on $w$. This completes the proof.

\section{Mappings That Take Values of Inner Product}

In this section, we investigate the relationship between the Möbius operations and the linear functional which takes the value of the inner product of each vector and a fixed vector. Then, a representation theorem of Riesz type is considered.

We need a well-known notion of continuity of mappings between metric spaces and a notation for asymptotic behavior of functions in elementary calculus.

Definition 13. Let $\left(X_{1}, d_{1}\right)$ and $\left(X_{2}, d_{2}\right)$ be two metric spaces. A map $f: X_{1} \longrightarrow X_{2}$ is said to be Lipschitz continuous if

$$
\sup _{x, y \in X_{1}, x \neq y} \frac{d_{2}(f(x), f(y))}{d_{1}(x, y)}<\infty
$$

holds. Then, the left-hand side of (52) is called the Lipschitz constant of $f$ and denoted by $L(f)$.

Definition 14. Let $g(s)$ be a real valued function of a real variable $s$. For any real constant $\alpha, g(s)=o\left(s^{\alpha}\right)(s \longrightarrow \infty)$ means that $g(s) / s^{\alpha} \longrightarrow 0$ as $s \longrightarrow \infty$. In particular, $g(s)=$ $o(1)(s \longrightarrow \infty)$ means that $g(s) \longrightarrow 0$ as $s \longrightarrow \infty$.

Theorem 15. Let $\mathbb{V}$ be a real inner product space, $\mathbf{c} \in \mathbb{V}$ with $\|\mathbf{c}\| \leq 1$, and consider the functional $f$ defined by the formula

$$
f(\mathbf{x})=\mathbf{x} \cdot \mathbf{c},
$$

for any $\mathbf{x} \in \mathbb{V}$. Then, 
(i) The restriction of $f$ to the Möbius gyrovector space $\mathbb{V}_{1}$ is Lipschitz continuous with the Lipschitz constant $\|\mathbf{c}\|$, if we consider the Poincaré metric on both the ball $\mathbb{V}_{1}$ and the interval $(-1,1)$

(ii) For any $\varepsilon>0$, the functional $f$ satisfies the following conditions:

$$
\begin{aligned}
-f(\mathbf{x}+\mathbf{y}) \oplus_{s} f\left(\mathbf{x} \oplus_{s} \mathbf{y}\right) & =o\left(s^{-2+\varepsilon}\right)(s \longrightarrow \infty), \\
-\{f(\mathbf{x})+f(\mathbf{y})\} \oplus_{s}\left\{f(\mathbf{x}) \oplus_{s} f(\mathbf{y})\right\} & =o\left(s^{-2+\varepsilon}\right)(s \longrightarrow \infty), \\
-f(r \mathbf{x}) \oplus_{s} f\left(r \otimes_{s} \mathbf{x}\right) & =o\left(s^{-2+\varepsilon}\right)(s \longrightarrow \infty), \\
-r f(\mathbf{x}) \oplus_{s} r \otimes_{s} f(\mathbf{x}) & =o\left(s^{-2+\varepsilon}\right)(s \longrightarrow \infty),
\end{aligned}
$$

for any $\mathbf{x}, \mathbf{y} \in \mathbb{V}$ and any $r \in \mathbb{R}$.

Proof. (i) For a while, let us denote the restriction of $f$ to $\mathbb{V}_{1}$ by $f$ simply. The Lipschitz continuity of $f$ is an immediate consequence of the Schwarz type inequality related to the Mobius operations. Actually, it follows from Theorem 8 that

$$
\left|\mathbf{x} \cdot \mathbf{c} \ominus_{1} \mathbf{y} \cdot \mathbf{c}\right| \leq\|\mathbf{c}\| \otimes_{1}\left\|\mathbf{x} \ominus_{1} \mathbf{y}\right\|
$$

or

$$
h(f(\mathbf{x}), f(\mathbf{y})) \leq\|\mathbf{c}\| h(\mathbf{x}, \mathbf{y})
$$

for any $\mathbf{x}, \mathbf{y} \in \mathbb{V}_{1}$, which shows

$$
L(f)=\sup _{\mathbf{x}, \mathbf{y} \in \mathbb{V}_{1}, \mathbf{x} \neq \mathbf{y}} \frac{h(f(\mathbf{x}), f(\mathbf{y}))}{h(\mathbf{x}, \mathbf{y})} \leq\|\mathbf{c}\| .
$$

On the other hand, put $c=L(f)$. Then, for any $\mathbf{x}, \mathbf{y} \in \mathbb{V}_{1}$, we have

$$
\begin{aligned}
\tanh ^{-1}\left|f(\mathbf{x}) \ominus_{1} f(\mathbf{y})\right| & =h(f(\mathbf{x}), f(\mathbf{y})) \leq \operatorname{ch}(\mathbf{x}, \mathbf{y}) \\
& =c \tanh ^{-1}\left\|\mathbf{x} \ominus_{1} \mathbf{y}\right\| .
\end{aligned}
$$

By taking $\mathbf{x}=\mathbf{c} / 2 s$ and $\mathbf{y}=\mathbf{0}$, we have

$$
\begin{aligned}
\frac{\|\mathbf{c}\|^{2}}{2 s} & =\left|f\left(\frac{\mathbf{c}}{2 s}\right)\right|=\left|f(\mathbf{x}) \ominus_{1} f(\mathbf{0})\right| \leq \tanh \left(c \tanh ^{-1}\left\|\mathbf{x} \ominus_{1} \mathbf{0}\right\|\right) \\
& =\tanh \left(c \tanh ^{-1}\left\|\frac{\mathbf{c}}{2 s}\right\|\right),
\end{aligned}
$$

which yields

$$
\frac{\|\mathbf{c}\|^{2}}{2} \leq s \tanh \left(c \tanh ^{-1} \frac{\|\mathbf{c} / 2\|}{s}\right) \longrightarrow c \frac{\|\mathbf{c}\|}{2}(s \longrightarrow \infty) .
$$

Thus, we can obtain $\|\mathbf{c}\| \leq c$.

(ii) For any $\mathbf{x}, \mathbf{y} \in \mathbb{V}$ and sufficiently large real number $s$, it is immediate to see that

$$
\begin{aligned}
-f(\mathbf{x}+\mathbf{y}) \oplus_{s} f\left(\mathbf{x} \oplus_{s} \mathbf{y}\right) & =-\{\mathbf{x} \cdot \mathbf{c}+\mathbf{y} \cdot \mathbf{c}\} \oplus_{s} \frac{\left(1+\left(2 / s^{2}\right) \mathbf{x} \cdot \mathbf{y}+\left(1 / s^{2}\right)\|\mathbf{y}\|^{2}\right) \mathbf{x} \cdot \mathbf{c}+\left(1-\left(1 / s^{2}\right)\|\mathbf{x}\|^{2}\right) \mathbf{y} \cdot \mathbf{c}}{1+\left(2 / s^{2}\right) \mathbf{x} \cdot \mathbf{y}+\left(1 / s^{4}\right)\|\mathbf{x}\|^{2}\|\mathbf{y}\|^{2}} \\
& =\frac{\left[-\{\mathbf{x} \cdot \mathbf{c}+\mathbf{y} \cdot \mathbf{c}\}\left\{1+\left(2 / s^{2}\right) \mathbf{x} \cdot \mathbf{y}+\left(1 / s^{4}\right)\|\mathbf{x}\|^{2}\|\mathbf{y}\|^{2}\right\}+\left(1+\left(2 / s^{2}\right) \mathbf{x} \cdot \mathbf{y}+\left(1 / s^{2}\right)\|\mathbf{y}\|^{2}\right) \mathbf{x} \cdot \mathbf{c}+\left(1-\left(1 / s^{2}\right)\|\mathbf{x}\|^{2}\right) \mathbf{y} \cdot \mathbf{c}\right]}{\{1+o(1)\}}
\end{aligned}
$$

and it is also straightforward to calculate the numerator of this formula as

$$
\begin{aligned}
& -\left\{\mathbf{x} \cdot \mathbf{c}+\frac{2}{s^{2}}(\mathbf{x} \cdot \mathbf{c})(\mathbf{x} \cdot \mathbf{y})+\frac{1}{s^{4}}\|\mathbf{x}\|^{2}\|\mathbf{y}\|^{2} \mathbf{x} \cdot \mathbf{c}+\mathbf{y} \cdot \mathbf{c}\right. \\
& \left.\quad+\frac{2}{s^{2}}(\mathbf{y} \cdot \mathbf{c})(\mathbf{x} \cdot \mathbf{y})+\frac{1}{s^{4}}\|\mathbf{x}\|^{2}\|\mathbf{y}\|^{2} \mathbf{y} \cdot \mathbf{c}\right\} \\
& \quad+\left\{\mathbf{x} \cdot \mathbf{c}+\frac{2}{s^{2}}(\mathbf{x} \cdot \mathbf{y})(\mathbf{x} \cdot \mathbf{c})+\frac{1}{s^{2}}\|\mathbf{y}\|^{2} \mathbf{x} \cdot \mathbf{c}+\mathbf{y} \cdot \mathbf{c}-\frac{1}{s^{2}}\|\mathbf{x}\|^{2} \mathbf{y} \cdot \mathbf{c}\right\} \\
& \quad=\frac{1}{s^{2}}\left\{-2(\mathbf{y} \cdot \mathbf{c})(\mathbf{x} \cdot \mathbf{y})+\|\mathbf{y}\|^{2} \mathbf{x} \cdot \mathbf{c}-\|\mathbf{x}\|^{2} \mathbf{y} \cdot \mathbf{c}\right\} \\
& \quad+\frac{1}{s^{4}}\left\{-\|\mathbf{x}\|^{2}\|\mathbf{y}\|^{2} \mathbf{x} \cdot \mathbf{c}-\|\mathbf{x}\|^{2}\|\mathbf{y}\|^{2} \mathbf{y} \cdot \mathbf{c}\right\},
\end{aligned}
$$

$$
\begin{aligned}
-\{ & f(\mathbf{x})+\mathrm{f}(\mathbf{y})\} \oplus_{s}\left\{f(\mathbf{x}) \oplus_{s} f(\mathbf{y})\right\} \\
& =-\{\mathbf{x} \cdot \mathbf{c}+\mathbf{y} \cdot \mathbf{c}\} \oplus_{s} \frac{\mathbf{x} \cdot \mathbf{c}+\mathbf{y} \cdot \mathbf{c}}{1+\left(1 / s^{2}\right)(\mathbf{x} \cdot \mathbf{c})(\mathbf{y} \cdot \mathbf{c})} \\
& =\frac{\left[-\{\mathbf{x} \cdot \mathbf{c}+\mathbf{y} \cdot \mathbf{c}\}\left\{1+\left(1 / s^{2}\right)(\mathbf{x} \cdot \mathbf{c})(\mathbf{y} \cdot \mathbf{c})\right\}+\mathbf{x} \cdot \mathbf{c}+\mathbf{y} \cdot \mathbf{c}\right]}{\{1+o(1)\}} \\
& =\frac{-\left(1 / s^{2}\right)(\mathbf{x} \cdot \mathbf{c}+\mathbf{y} \cdot \mathbf{c})(\mathbf{x} \cdot \mathbf{c})(\mathbf{y} \cdot \mathbf{c})}{\{1+o(1)\}},
\end{aligned}
$$

which yields the second formula of (ii). For any $\varepsilon>0$, real numbers $r$ and $x$, it is elementary to check

$s \tanh \left(r \tanh ^{-1} \frac{x}{s}\right)=r x+\frac{r\left(1-r^{2}\right) x^{3}}{3 s^{2}}+o\left(s^{-4+\varepsilon}\right)(s \longrightarrow \infty)$.

which yields the first formula of (ii). Next, 
We can obtain

$-f(r \mathbf{x}) \oplus_{s} f\left(r \otimes_{s} \mathbf{x}\right)=\frac{\left\{-r \mathbf{x} \cdot \mathbf{c}+s \tanh \left(r \tanh ^{-1}(\|\mathbf{x}\| / s)\right)(\mathbf{x} /\|\mathbf{x}\|) \cdot \mathbf{c}\right\}}{\{1+o(1)\}}$.

By formula (64), the numerator of this formula can be calculated as

$$
\begin{aligned}
& -r(\mathbf{x} \cdot \mathbf{c})+\left\{r\|\mathbf{x}\|+\frac{1}{3 s^{2}} r\left(1-r^{2}\right)\|\mathbf{x}\|^{3}+o\left(s^{-4+\varepsilon}\right)\right\} \frac{\mathbf{x}}{\|\mathbf{x}\|} \cdot \mathbf{c} \\
& =\frac{1}{3 s^{2}} r\left(1-r^{2}\right)(\mathbf{x} \cdot \mathbf{c})\|\mathbf{x}\|^{2}+o\left(s^{-4+\varepsilon}\right),
\end{aligned}
$$

which shows the third formula of (ii). We also obtain

$-r f(\mathbf{x}) \oplus_{s} r \otimes_{s} f(\mathbf{x})=\frac{\left\{-r \mathbf{x} \cdot \mathbf{c}+s \tanh \left(r \tanh ^{-1}((\mathbf{x} \cdot \mathbf{c}) / s)\right)\right\}}{\{1+o(1)\}}$.

The numerator of this formula can be calculated as

$$
\begin{aligned}
& -r(\mathbf{x} \cdot \mathbf{c})+\left\{r(\mathbf{x} \cdot \mathbf{c})+\frac{1}{3 s^{2}} r\left(1-r^{2}\right)(\mathbf{x} \cdot \mathbf{c})^{3}+o\left(s^{-4+\varepsilon}\right)\right\} \\
& \quad=\frac{1}{3 s^{2}} r\left(1-r^{2}\right)(\mathbf{x} \cdot \mathbf{c})^{3}+o\left(s^{-4+\varepsilon}\right),
\end{aligned}
$$

which shows the fourth formula of (ii). This completes the proof.

In the rest of this section, we consider a representation theorem of Riesz type. Theorem 11 shows that, in a certain sense, the gyroadditivity (31) is too much strong for continuous functionals. Therefore, it is natural to introduce a suitable notion for functionals on the Möbius gyrovector spaces which is corresponding to the linearity of functionals on inner product spaces.

For any general (not necessarily linear) mapping $f$ from the Möbius gyrovector space $\mathbb{V}_{1}$ to the interval $(-1,1)$, we associate a family $\left\{f_{s}\right\}$ of mappings defined as follows.

Definition 16. Let $\mathbb{V}$ be a real inner product space. For any mapping $f: \mathbb{V}_{1} \longrightarrow(-1,1)$ and any positive real number $s$, we define a map $f_{s}: \mathbb{V}_{s} \longrightarrow(-s, s)$ by

$$
f_{s}(\mathbf{x})=s f\left(\frac{\mathbf{x}}{s}\right)
$$

for any element $\mathbf{x} \in \mathbb{V}_{s}$.

It seems that Theorem 15 provides sufficiently reasonable motivation for making the following definitions.

Definition 17. (i) Let $\mathbf{c} \in \mathbb{V}$ and $\|\mathbf{c}\| \leq 1$. A mapping $f: \mathbb{V}_{1}$ $\longrightarrow(-1,1)$ is said to be quasi gyrolinear with respect to $\mathbf{c}$ if the family $\left\{f_{s}\right\}$ defined by formula (69) satisfies the following conditions:

$$
\begin{gathered}
f_{s}\left(\mathbf{x} \oplus_{s} \mathbf{y}\right) \longrightarrow(\mathbf{x}+\mathbf{y}) \cdot \mathbf{c}(s \longrightarrow \mathbf{\infty}), \\
f_{s}(\mathbf{x}) \oplus_{s} f_{s}(\mathbf{y}) \longrightarrow \mathbf{x} \cdot \mathbf{c}+\mathbf{y} \cdot \mathbf{c}(s \longrightarrow \infty), \\
f_{s}\left(r \otimes_{s} \mathbf{x}\right) \longrightarrow(r \mathbf{x}) \cdot \mathbf{c}(s \longrightarrow \infty), \\
r \otimes_{s} f_{s}(\mathbf{x}) \longrightarrow r(\mathbf{x} \cdot \mathbf{c})(s \longrightarrow \infty),
\end{gathered}
$$

for any element $\mathbf{x}, \mathbf{y} \in \mathbb{V}$ and any real number $r \in \mathbb{R}$.

(ii) A mapping $f: \mathbb{V}_{1} \longrightarrow(-1,1)$ is said to be asymptotically gyrolinear if the family $\left\{f_{s}\right\}$ defined by formula (69) satisfies the following conditions:

$$
\begin{array}{r}
-\left\{f_{s}(\mathbf{x}) \oplus_{s} f_{s}(\mathbf{y})\right\} \oplus_{s} f_{s}\left(\mathbf{x} \oplus_{s} \mathbf{y}\right) \longrightarrow 0(s \longrightarrow \infty), \\
-\left\{r \otimes_{s} f_{s}(\mathbf{x})\right\} \oplus_{s} f_{s}\left(r \otimes_{s} \mathbf{x}\right) \longrightarrow 0(s \longrightarrow \infty),
\end{array}
$$

for any element $\mathbf{x}, \mathbf{y} \in \mathbb{V}$ and any real number $r \in \mathbb{R}$.

Remark 18. Let $\mathbf{c} \in \mathbb{V}$ and $\|\mathbf{c}\| \leq 1$. (i) Every quasi gyrolinear map from $\mathbb{V}_{1}$ to $(-1,1)$ with respect to $\mathbf{c}$ is asymptotically gyrolinear. It is easy to check by using Lemma 5 .

(ii) The restriction of the mapping defined by formula (53) to the Möbius gyrovector space $\mathbb{V}_{1}$ is quasi gyrolinear by Theorem 15(ii).

The following lemma can be verified immediately by the definition of the Möbius addition $\oplus_{s}$, so we omit the proof.

Lemma 19. Suppose that $\mathbf{u}(s), \mathbf{v}(s)$ are elements in $\mathbb{V}_{s}$ defined for sufficiently large real number such that $\mathbf{u}(s), \mathbf{v}(s)$ converge to constant vectors $\mathbf{a}, \mathbf{b}$ as $s \longrightarrow \infty$, respectively. Then, $\mathbf{u}(s) \oplus_{s} \mathbf{v}(s) \longrightarrow \mathbf{a}+\mathbf{b}$ as $s \longrightarrow \infty$.

Although we already know that $r \otimes_{s} a \longrightarrow r a$ as $s \longrightarrow \infty$ for any constants $r, a \in \mathbb{R}$, we need a lemma for the case where $a$ is replaced by a function $g(s)$ which converges to a constant as $s \longrightarrow \infty$.

Lemma 20. There exists a function $C(r)$ which depends on $r$ and the formula

$$
\left|r \otimes_{1} x-r x\right| \leq C(r)|x|^{3}\left(-\frac{1}{2} \leq x \leq \frac{1}{2}\right)
$$

holds.

Proof. For a while, assume $r>0$. Put $f(x)=r \otimes_{1} x=\tanh$ $\left(r \tanh ^{-1} x\right)$. For any $x \in(-1,1)$, it follows from Maclaurin's theorem that there exists a number $0<\theta<1$ satisfying the equation

$$
f(x)=f(0)+f^{\prime}(0) x+\frac{f^{\prime \prime}(\theta x)}{2 !} x^{2}
$$


It is elementary to see

$$
\begin{aligned}
& f^{\prime}(x)=\left\{1-\left(\tanh \left(r \tanh ^{-1} x\right)\right)^{2}\right\} \frac{r}{1-x^{2}} f^{\prime}(0)=r, \\
& f^{\prime \prime}(x)=\left\{1-\left(\tanh \left(r \tanh ^{-1} x\right)\right)^{2}\right\} \frac{2 r}{\left(1-x^{2}\right)^{2}}\left[-r\left(\tanh \left(r \tanh ^{-1} x\right)\right)+x\right],
\end{aligned}
$$

so that we obtain

$$
\begin{gathered}
\left|r \otimes_{1} x-r x\right|=\left|\frac{f^{\prime \prime}(\theta x)}{2 !} x^{2}\right|=\mid\left\{1-\left(\tanh \left(r \tanh ^{-1} \theta x\right)\right)^{2}\right\} \\
\left.\cdot \frac{r}{\left(1-(\theta x)^{2}\right)^{2}}\left[-r\left(\tanh \left(r \tanh ^{-1} \theta x\right)\right)+\theta x\right]|| x\right|^{2} .
\end{gathered}
$$

Now we restrict $|x| \leq 1 / 2$. If $0<r<1$, then we have $\left|r \otimes_{1} \theta x\right|=r \otimes_{1}|\theta x|<|\theta x|<|x|$, which yields

$$
\left|r \otimes_{1} x-r x\right| \leq \frac{16}{9} r(r+1)|x|^{3} .
$$

If $r>1$, then we have $\left|r \otimes_{1} \theta x\right|=r \otimes_{1}|\theta x|<r|\theta x|<r|x|$, which yields

$$
\left|r \otimes_{1} x-r x\right| \leq \frac{16}{9} r\left(r^{2}+1\right)|x|^{3} .
$$

For $r<0$, since we have the relation $\left|r \otimes_{1} x-r x\right|=$ $\left|(-r) \otimes_{1} x-(-r) x\right|$, a similar argument shows the conclusion. This completes the proof.

Lemma 21. Let $r, a \in \mathbb{R}$. Suppose that $g(s)$ is a real valued function defined for sufficiently large real number $s$ which satisfies the condition $g(s) \longrightarrow a$ as $s \longrightarrow \infty$. Then, $r \otimes_{s} g(s)$ $\longrightarrow$ ra as $s \longrightarrow \infty$.

Proof. For any $x \in \mathbb{R}$, if we take $s \geq 2|x|$, then obviously $-1 / 2$ $\leq x / s \leq 1 / 2$. It follows from Lemma 20 that

$$
\begin{gathered}
\left|r \otimes_{1} \frac{x}{s}-r \frac{x}{s}\right| \leq C(r)\left|\frac{x}{s}\right|^{3}, \\
\left|r \otimes_{s} x-r x\right| \leq C(r) \frac{|x|^{3}}{s^{2}} .
\end{gathered}
$$

Therefore, for sufficiently large $s>0$, we obtain

$$
\left|r \otimes_{s} g(s)-r g(s)\right| \leq C(r) \frac{|g(s)|^{3}}{s^{2}}
$$

and hence,

$$
\begin{aligned}
\left|r \otimes_{s} g(s)-r a\right| & \leq\left|r \otimes_{s} g(s)-r g(s)\right|+|r g(s)-r a| \\
& \leq C(r) \frac{|g(s)|^{3}}{s^{2}}+|r||g(s)-a| \longrightarrow 0
\end{aligned}
$$

as $s \longrightarrow \infty$. This completes the proof.

The following result can be considered as a representation theorem of Riesz type in the Möbius gyrovector space.

Theorem 22. Let $\mathbb{V}$ be a real Hilbert space. Suppose that $f: \mathbb{V}_{1} \longrightarrow(-1,1)$ is asymptotically gyrolinear and Lipschitz continuous with $L(f) \leq 1$ considering the Poincaré metrics on both the Möbius gyrovector space $\mathbb{V}_{1}$ and the interval $(-1,1)$. Additionally, assume that $f$ satisfies the following condition:

For any element $\mathbf{x} \in \mathbb{V}, \lim _{s \longrightarrow \infty} f_{s}(\mathbf{x})$ exists as a real number

Then, there exists a unique element $\mathbf{c} \in \mathbb{V}$ satisfying $\|\mathbf{c}\| \leq L(f)$ and $f$ is quasi gyrolinear with respect to $\mathbf{c}$.

Proof. Put $c=L(f)$. Note that it implies

$$
\left|f(\mathbf{x}) \ominus_{1} f(\mathbf{y})\right| \leq c \otimes_{1}\left\|\mathbf{x} \ominus_{1} \mathbf{y}\right\|,
$$

for any $\mathbf{x}, \mathbf{y} \in \mathbb{V}_{1}$. For any $\mathbf{x}, \mathbf{y} \in \mathbb{V}$ and any sufficiently large $s>0$, we have

$$
\begin{gathered}
\frac{\left|f_{s}(\mathbf{x}) \ominus_{s} f_{s}(\mathbf{y})\right|}{s}=\left|\frac{f_{s}(\mathbf{x})}{s} \ominus_{1} \frac{f_{s}(\mathbf{y})}{s}\right|=\left|f\left(\frac{\mathbf{x}}{s}\right) \ominus_{1} f\left(\frac{\mathbf{y}}{s}\right)\right| \\
\leq c \otimes_{1}\left\|\frac{\mathbf{x}}{s} \ominus_{1} \frac{\mathbf{y}}{s}\right\|=c \otimes_{1} \frac{\left\|\mathbf{x} \ominus_{s} \mathbf{y}\right\|}{s}=\frac{c \otimes_{s}\left\|\mathbf{x} \ominus_{s} \mathbf{y}\right\|}{s},
\end{gathered}
$$

which yields

$$
\left|f_{s}(\mathbf{x}) \ominus_{s} f_{s}(\mathbf{y})\right| \leq c \otimes_{s}\left\|\mathbf{x} \ominus_{s} \mathbf{y}\right\| .
$$

Now, it follows from Lemma 6(ii) that

$$
\begin{gathered}
\left\|\left(\mathbf{x} \oplus_{s} \mathbf{y}\right) \ominus_{s}(\mathbf{x}+\mathbf{y})\right\| \leq 2\left\|\left(\mathbf{x} \oplus_{s} \mathbf{y}\right)-(\mathbf{x}+\mathbf{y})\right\| \longrightarrow 0(s \longrightarrow \infty), \\
\left\|\left(r \otimes_{s} \mathbf{x}\right) \ominus_{s}(r \mathbf{x})\right\| \leq 2\left\|\left(r \otimes_{s} \mathbf{x}\right)-(r \mathbf{x})\right\| \longrightarrow 0(s \longrightarrow \infty) .
\end{gathered}
$$

Therefore, by inequality (88) and Lemma 21 , we can obtain

$$
\begin{aligned}
& \left|f_{s}\left(\mathbf{x} \oplus_{s} \mathbf{y}\right) \ominus_{s} f_{s}(\mathbf{x}+\mathbf{y})\right| \\
& \quad \leq c \otimes_{s}\left\|\left(\mathbf{x} \oplus_{s} \mathbf{y}\right) \ominus_{s}(\mathbf{x}+\mathbf{y})\right\| \longrightarrow 0(s \longrightarrow \infty), \\
& \left|f_{s}\left(r \otimes_{s} \mathbf{x}\right) \ominus_{s} f_{s}(r \mathbf{x})\right| \\
& \quad \leq c \otimes_{s}\left\|\left(r \otimes_{s} \mathbf{x}\right) \ominus_{s}(r \mathbf{x})\right\| \longrightarrow 0(s \longrightarrow \infty),
\end{aligned}
$$


for any $\mathbf{x}, \mathbf{y} \in \mathbb{V}$ and $r \in \mathbb{R}$. Define a functional $F: \mathbb{V} \longrightarrow \mathbb{R}$ by the formula

$$
F(\boldsymbol{x})=\lim _{s \rightarrow \infty} f_{s}(\boldsymbol{x}),
$$

for any $\mathbf{x} \in \mathbb{V}$.

By Lemma 19, formulae (74) and (90), for any $\varepsilon>0$, there exists a real number $s_{0}>0$ such that, if $s \geq s_{0}$ then

$$
\begin{array}{r}
\left|-\{F(\mathbf{x})+F(\mathbf{y})\}+\left\{f_{s}(\mathbf{x}) \oplus_{s} f_{s}(\mathbf{y})\right\}\right|<\varepsilon, \\
\left|-\left\{f_{s}(\mathbf{x}) \oplus_{s} f_{s}(\mathbf{y})\right\} \oplus_{s} f_{s}\left(\mathbf{x} \oplus_{s} \mathbf{y}\right)\right|<\varepsilon, \\
\left|f_{s}\left(\mathbf{x} \oplus_{s} \mathbf{y}\right) \ominus_{s} f_{s}(\mathbf{x}+\mathbf{y})\right|<\varepsilon, \\
\left|f_{s}(\mathbf{x}+\mathbf{y})-F(\mathbf{x}+\mathbf{y})\right|<\varepsilon .
\end{array}
$$

We can estimate

$$
\begin{aligned}
\mid-\{ & F(\mathbf{x})+F(\mathbf{y})\}+F(\mathbf{x}+\mathbf{y})|\leq|-\{F(\mathbf{x})+F(\mathbf{y})\} \\
& +\left\{f_{s}(\mathbf{x}) \oplus_{s} f_{s}(\mathbf{y})\right\}|+|-\left\{f_{s}(\mathbf{x}) \oplus_{s} f_{s}(\mathbf{y})\right\}+f_{s}\left(\mathbf{x} \oplus_{s} \mathbf{y}\right) \mid \\
& +\left|-f_{s}\left(\mathbf{x} \oplus_{s} \mathbf{y}\right)+f_{s}(\mathbf{x}+\mathbf{y})\right|+\left|-f_{s}(\mathbf{x}+\mathbf{y})+F(\mathbf{x}+\mathbf{y})\right| \\
& \leq\left|-\{F(\mathbf{x})+F(\mathbf{y})\}+\left\{f_{s}(\mathbf{x}) \oplus_{s} f_{s}(\mathbf{y})\right\}\right| \\
& +2\left|-\left\{f_{s}(\mathbf{x}) \oplus_{s} f_{s}(\mathbf{y})\right\} \oplus_{s} f_{s}\left(\mathbf{x} \oplus_{s} \mathbf{y}\right)\right| \\
& +2\left|f_{s}\left(\mathbf{x} \oplus_{s} \mathbf{y}\right) \Theta_{s} f_{s}(\mathbf{x}+\mathbf{y})\right|+\mid-f_{s}(\mathbf{x}+\mathbf{y}) \\
& +F(\mathbf{x}+\mathbf{y}) \mid<6 \varepsilon
\end{aligned}
$$

for any $s \geq s_{0}$, which implies $F(\mathbf{x}+\mathbf{y})=F(\mathbf{x})+F(\mathbf{y})$.

Similarly, by Lemma 21 , formulae (75), (91), and (88), we can obtain the formulae

$$
\begin{aligned}
F(r \mathbf{x}) & =r F(\mathbf{x}), \\
|F(\mathbf{x})-F(\mathbf{y})| & \leq c\|\mathbf{x}-\mathbf{y}\|,
\end{aligned}
$$

for any $\mathbf{x}, \mathbf{y} \in \mathbb{V}$ and $r \in \mathbb{R}$. Thus, $F: \mathbb{V} \longrightarrow \mathbb{R}$ is a bounded linear functional which is defined on the whole space $\mathbb{V}$ and $\|F\| \leq c$. It follows from the classical Riesz theorem that there exists a unique element $\mathbf{c} \in \mathbb{V}$ satisfying $F(\mathbf{x})=\mathbf{x} \cdot \mathbf{c}$ for any $\mathbf{x} \in \mathbb{V}$ and $\|\mathbf{c}\|=\|F\|$. Moreover, it is easy to verify formulae (70)-(73) by using formula (90), formula (91), Lemma 19, and Lemma 20. Thus, $f$ is quasi gyrolinear with respect to c. This completes the proof.

Remark 23. Instead of condition (85), if we assume that there exists a positive homogeneous map $\tilde{f}: \mathbb{V} \longrightarrow \mathbb{R}$ which is an extension of $f$, then there exists a unique element $\mathbf{c} \in \mathbb{V}$ satisfying

$$
\tilde{f}(\mathbf{x})=\mathbf{x} \cdot \mathbf{c},
$$

for any $\mathbf{x} \in \mathbb{V}$.

\section{Continuous Quasi Gyrolinear Functionals}

In this section, we present a class of continuous functionals on Möbius gyrovector spaces, which consists of a counterpart to continuous linear functionals on real Hilbert spaces.
Lemma 24. Let $\left\{c_{j}\right\}_{j=1}^{\infty}$ and $\left\{a_{j}\right\}_{j=1}^{\infty}$ be square summable sequences of real numbers. Note that

$$
\text { we do not assume } \sum_{j=1}^{\infty} c_{j}^{2} \leq 1 \text { or } \sum_{j=1}^{\infty} a_{j}^{2} \leq 1 \text {. }
$$

Suppose that $s_{0}$ is a positive real number and $u_{j}(s)$ is a real valued function defined for $s \geq s_{0}$ and $j=1,2, \cdots$, which satisfies the following conditions:

$$
\begin{array}{cc}
-s<u_{j}(s)<s, & \text { for } s \geq s_{0} \text { and } j=1,2, \cdots, \\
u_{j}(s) \longrightarrow a_{j}, & \text { as } s \longrightarrow \infty \text { for any } j=1,2, \cdots, \\
\sum_{j=1}^{\infty} \boldsymbol{\oplus}_{s^{2}} u_{j}(s)^{2} \leq K, & \text { for } s \geq s_{0},
\end{array}
$$

where $K$ is a constant which does not depend on s. Then, the series $\sum_{j=1}^{\infty} \oplus_{s} c_{j} \otimes_{s} u_{j}(s)$ converges for any $s \geq s_{0}$, and

$$
\sum_{j=1}^{\infty}{ }^{\oplus_{s}} c_{j} \otimes_{s} u_{j}(s) \longrightarrow \sum_{j=1}^{\infty} c_{j} a_{j}
$$

as $s \longrightarrow \infty$.

Proof. For any $s \geq s_{0}$, any natural number $n$ and $p$, we can apply the gyrotriangle inequality and a Cauchy type inequality ([12], Remark 2.6) to obtain

$$
\begin{aligned}
& \left|-\sum_{j=1}^{n} c_{j}^{\oplus_{s}} \otimes_{s} u_{j}(s) \oplus_{s} \sum_{j=1}^{n+p^{\oplus_{s}}} c_{j} \otimes_{s} u_{j}(s)\right|=\left|\sum_{j=n+1}^{n+p} c_{j}^{\oplus_{s}} \otimes_{s} u_{j}(s)\right| \\
& \quad \leq \sum_{j=n+1}^{n+p} \oplus_{s}\left|c_{j} \otimes_{s} u_{j}(s)\right|=\sum_{j=n+1}^{n+p}\left(\left|c_{j}\right| \otimes_{s}\left|u_{j}(s)\right|\right) \\
& \quad \leq\left(\sum_{j=n+1}^{\infty} c_{j}^{2}\right)^{1 / 2} \otimes_{s}\left(\sum_{j=n+1}^{\infty} u_{j}^{\oplus_{s}^{2}} u_{j}(s)^{2}\right)^{1 / 2},
\end{aligned}
$$

which yields that the sequence $\left\{\sum_{j=1}^{n} \oplus_{s} c_{j} \otimes_{s} u_{j}(s)\right\}_{n=1}^{\infty}$ is a Cauchy sequence in the interval $((-s, s), h)$ (cf. [11], Lemma 20(ii)), and hence, it converges.

From the proof of Lemma 20, if $0<r<1$, then the inequality

$$
\left|r \otimes_{1} x-r x\right| \leq 4 r|x|^{3}\left(-\frac{1}{2} \leq x \leq \frac{1}{2}\right)
$$

holds. By putting $s_{1}=2 K^{1 / 2}+1$, a similar argument to the proof of Lemma 21 shows that

$$
\begin{aligned}
\left|r \otimes_{s} K^{1 / 2}-r K^{1 / 2}\right| & \leq 4 r \frac{K^{3 / 2}}{s^{2}}, \\
0 & \leq r \otimes_{s} K^{1 / 2} \leq r K^{1 / 2}+4 r K^{3 / 2},
\end{aligned}
$$


for any $s \geq s_{1}$. Therefore, for any positive number $\varepsilon$, there exists a natural number $j_{0}$ such that

$$
\begin{array}{r}
\sum_{j=j_{0}+1}^{\infty}\left|c_{j} a_{j}\right|<\varepsilon, \\
\sup _{s \geq s_{1}}\left[\left(\sum_{j=j_{0}+1}^{\infty} c_{j}^{2}\right)^{1 / 2} \otimes_{s} K^{1 / 2}\right]<\varepsilon .
\end{array}
$$

Moreover, we can take a positive number $s_{2}$ such that

$$
\left|\sum_{j=1}^{j_{0}} c_{j} a_{j}-\sum_{j=1}^{j_{0} \oplus_{s}} c_{j} \otimes_{s} u_{j}(s)\right|<\varepsilon,
$$

for any $s>s_{2}$. Then, by [11], Lemma 14(iii), and [12], Remark 2.6, we can obtain

$$
\begin{aligned}
& \left|\sum_{j=1}^{\infty} c_{j} a_{j}-\sum_{j=1}^{\infty} \oplus_{s} c_{j} \otimes_{s} u_{j}(s)\right| \leq\left|\sum_{j=1}^{\infty} c_{j} a_{j}-\sum_{j=1}^{j_{0}} c_{j} a_{j}\right| \\
& +\left|\sum_{j=1}^{j_{0}} c_{j} a_{j}-\sum_{j=1}^{j_{0} \oplus_{s}} c_{j} \otimes_{s} u_{j}(s)\right| \\
& +\left|\sum_{j=1}^{j_{0} \oplus_{s}} c_{j} \otimes_{s} u_{j}(s)-\sum_{j=1}^{\infty} c_{j} \otimes_{s} u_{j}(s)\right| \leq \sum_{j=j_{0}+1}^{\infty}\left|c_{j} a_{j}\right| \\
& +\left|\sum_{j=1}^{j_{0}} c_{j} a_{j}-\sum_{j=1}^{j_{0} \oplus_{s}} c_{j} \otimes_{s} u_{j}(s)\right| \\
& +2\left|-\sum_{j=1}^{j_{0} \oplus_{s}} c_{j} \otimes_{s} u_{j}(s) \oplus_{s} \sum_{j=1}^{\infty} \oplus_{s} \otimes_{s} u_{j}(s)\right|<2 \varepsilon \\
& +2\left|\sum_{j=j_{0}+1}^{\infty} c_{j} \otimes_{s} u_{j}(s)\right| \leq 2 \varepsilon \\
& +2\left[\left(\sum_{j=j_{0}+1}^{\infty} c_{j}^{2}\right)^{1 / 2} \otimes_{s}\left(\sum_{j=j_{0}+1}^{\infty} u_{j}^{\oplus_{s}} u_{j}(s)^{2}\right]<4 \varepsilon\right.
\end{aligned}
$$

for sufficiently large $s$. This completes the proof.

Theorem 25. Let $\left\{\mathbf{e}_{j}\right\}_{j=1}^{\infty}$ be a complete orthonormal sequence in a real Hilbert space $V$ and let $\mathbf{x}$ be an arbitrary element in $\mathbb{V}_{1}$. We can apply the orthogonal gyroexpansion ([11], Theorem 35) to get a unique sequence $\left(r_{1}, r_{2}, \cdots\right)$ of real numbers such that

$$
\mathbf{x}=\sum_{j=1}^{\infty \oplus_{1}} r_{j} \otimes_{1} \frac{e_{j}}{2}
$$

Then, the following inequality holds:

$$
\left(\sum_{j=1}^{\infty} r_{j}^{2}\right)^{1 / 2} \leq \frac{\tanh ^{-1}\|\boldsymbol{x}\|}{\tanh ^{-1}(1 / 2)} .
$$

Proof. Note that

$$
\|\mathbf{x}\|^{2}=\sum_{j=1}^{\infty \oplus_{1}}\left(r_{j} \otimes_{1} \frac{1}{2}\right)^{2}
$$

by [11], Theorem 36(iii). Let $\left\{c_{j}\right\}_{j=1}^{\infty}$ be an arbitrary square summable sequence of real numbers. Then, for any natural number $n$, by identities of Lemma 5 and a Cauchy type inequality ([12], Theorem 2.5), we obtain the following estimation:

$$
\begin{aligned}
& \left(\sum_{j=1}^{n}\left|c_{j} r_{j}\right|\right) \otimes_{1} \frac{1}{2}=\sum_{j=1}^{n \oplus_{1}}\left(\left|c_{j} r_{j}\right| \otimes_{1} \frac{1}{2}\right)=\sum_{j=1}^{n \oplus_{1}}\left(\left|c_{j}\right| \otimes_{1}\left|r_{j} \otimes_{1} \frac{1}{2}\right|\right) \\
& \leq\left(\sum_{j=1}^{n} c_{j}^{2}\right)^{1 / 2} \otimes_{1}\left(\sum_{j=1}^{n \oplus_{1}}\left(r_{j} \otimes_{1} \frac{1}{2}\right)^{2}\right)^{1 / 2} .
\end{aligned}
$$

Thus, we obtain

$$
\left(\sum_{j=1}^{\infty}\left|c_{j} r_{j}\right|\right) \otimes_{1} \frac{1}{2} \leq\|\mathbf{c}\| \otimes_{1}\|\mathbf{x}\|
$$

or

$$
\sum_{j=1}^{\infty}\left|c_{j} r_{j}\right| \leq\|\boldsymbol{c}\| \frac{\tanh ^{-1}\|\mathbf{x}\|}{\tanh ^{-1}(1 / 2)}
$$

where $\mathbf{c}$ is a unique element in $\mathbb{V}$ defined by the formula $\mathbf{c}=\sum_{j=1}^{\infty} c_{j} \mathbf{e}_{j}$. Because $\left\{c_{j}\right\}$ is an arbitrary square summable sequence, we can conclude inequality (107). This completes the proof.

We show that, if a sequence $\left\{\mathbf{x}_{k}\right\}_{k}$ in a Möbius gyrovector space converges to an element $\mathbf{x}$, then the $j$ th gyrocoefficient of $\mathbf{x}_{k}$ converges to that of $\mathbf{x}$.

Lemma 26. Let $\left\{\mathbf{e}_{j}\right\}_{j=1}^{\infty}$ be a complete orthonormal sequence in a real Hilbert space $\mathbb{V}$. Suppose that $\left\{\mathbf{x}_{k}\right\}_{k}$ is a sequence and $\mathbf{x}$ is an element in $\mathbb{V}_{1}$ such that $h\left(\boldsymbol{x}_{k}, \boldsymbol{x}\right) \longrightarrow 0$ as $k \longrightarrow \infty$. Let

$$
\begin{aligned}
\mathbf{x}_{k} & =\sum_{j=1}^{\infty \oplus_{1}} r_{k, j} \otimes_{1} \frac{\mathbf{e}_{j}}{2}, \\
\mathbf{x} & =\sum_{j=1}^{\infty} r_{j}^{\oplus_{1}} \otimes_{1} \frac{\mathbf{e}_{j}}{2}
\end{aligned}
$$


be the orthogonal gyroexpansions. Then,

$$
r_{k, j} \longrightarrow r_{j}(k \longrightarrow \infty)
$$

for any $j=1,2, \cdots$.

Proof. Note that $\left\|\mathbf{x}_{k}-\mathbf{x}\right\| \longrightarrow 0$ as $k \longrightarrow \infty$ (cf. [11], Lemma 14(iii), Lemma 20). A similar argument on formula (118) in the proof of [11], Theorem 35, shows that

$$
\begin{gathered}
r_{k, 1}=\frac{\tanh ^{-1} \lambda_{k, 1}^{(1)}\left(\mathbf{x}_{k} \cdot \mathbf{e}_{1}\right)}{\tanh ^{-1}(1 / 2)}, \\
r_{1}=\frac{\tanh ^{-1} \lambda_{1}^{(1)}\left(\mathbf{x} \cdot \mathbf{e}_{1}\right)}{\tanh ^{-1}(1 / 2)}
\end{gathered}
$$

where

$$
\begin{aligned}
& \lambda_{k, 1}^{(1)}=\frac{\left\|\mathbf{x}_{k}\right\|^{2}+1-\sqrt{\left(\left\|\mathbf{x}_{k}\right\|^{2}+1\right)^{2}-4\left(\mathbf{x}_{k} \cdot \mathbf{e}_{1}\right)^{2}}}{2\left(\mathbf{x}_{k} \cdot \mathbf{e}_{1}\right)^{2}}, \\
& \lambda_{1}^{(1)}=\frac{\|\mathbf{x}\|^{2}+1-\sqrt{\left(\|\mathbf{x}\|^{2}+1\right)^{2}-4\left(\mathbf{x} \cdot \mathbf{e}_{1}\right)^{2}}}{2\left(\mathbf{x} \cdot \mathbf{e}_{1}\right)^{2}},
\end{aligned}
$$

which obviously yields $r_{k, 1} \longrightarrow r_{1}$ as $k \longrightarrow \infty$. Applying a similar argument to

$$
\begin{aligned}
-r_{k, 1} \otimes_{1} \frac{\mathbf{e}_{1}}{2} \oplus_{1} \mathbf{x}_{k} & =\sum_{j=2}^{\infty} r_{k, j} \otimes_{1} \frac{\mathbf{e}_{j}}{2}, \\
-r_{1} \otimes_{1} \frac{\mathbf{e}_{1}}{2} \oplus_{1} \mathbf{x} & =\sum_{j=2}^{\infty} r_{j} \otimes_{1} \frac{\mathbf{e}_{j}}{2},
\end{aligned}
$$

we obtain $r_{k, 2} \longrightarrow r_{2}$ as $k \longrightarrow \infty$, and so on. This completes the proof.

We present a class of continuous functionals on the Möbius gyrovector spaces, which seems to be a counterpart of bounded linear functionals on real Hilbert spaces.

Theorem 27. Let $\mathbb{V}$ be a real Hilbert space, let $\left\{\mathbf{e}_{j}\right\}_{j=1}^{\infty}$ be a complete orthonormal sequence in $\mathbb{V}$ and let $\left\{c_{j}\right\}_{j=1}^{\infty}$ be a square summable sequence of real numbers. Consider the Poincaré metrics on both the Möbius gyrovector space $\mathbb{V}_{1}$ and the interval $(-1,1)$. Then, we can define a map $f: \mathbb{V}_{1}$ $\longrightarrow(-1,1)$ by the equation

$$
f(\mathbf{x})=\left(\sum_{j=1}^{\infty} c_{j} r_{j}\right) \otimes_{1} \frac{1}{2}
$$

for any $\mathbf{x} \in \mathbb{V}_{1}$ whose orthogonal gyroexpansion is given by formula (106). Moreover, $f$ is continuous and quasi gyrolinear with respect to $\mathbf{c}=\sum_{j=1}^{\infty} c_{j} \mathbf{e}_{j}$.
Proof. Since the sequence $\left\{r_{j}\right\}_{j}$ is square summable by Theorem 25 , the ordinary infinite series $\sum_{j=1}^{\infty} c_{j} r_{j}$ converges absolutely; hence, the map $f$ is well defined. Suppose that $\left\{\mathbf{x}_{k}\right\}_{k}$ is a sequence and $\mathbf{x}$ is an element in $\mathbb{V}_{1}$ such that $h\left(\mathbf{x}_{k}, \mathbf{x}\right)$ $\longrightarrow 0$ as $k \longrightarrow \infty$. Let

$$
\begin{aligned}
\mathbf{x}_{k} & =\sum_{j=1}^{\infty} r_{k, j} \otimes_{1} \frac{\mathbf{e}_{j}}{2}, \\
\mathbf{x} & =\sum_{j=1}^{\infty} r_{j} \otimes_{1} \frac{\mathbf{e}_{j}}{2}
\end{aligned}
$$

be the orthogonal gyroexpansions. By Theorem 25,

$$
\left(\sum_{j=1}^{\infty} r_{k, j}^{2}\right)^{1 / 2} \leq \frac{\tanh ^{-1}\left\|\mathbf{x}_{k}\right\|}{\tanh ^{-1}(1 / 2)}
$$

which is bounded with respect to $k$, because $\left\|\mathbf{x}_{k}\right\| \longrightarrow\|\mathbf{x}\|<1$. Therefore, for any positive number $\varepsilon$, there exists a natural number $j_{0}$ such that

$$
\sum_{j=j_{0}+1}^{\infty}\left|c_{j} r_{k, j}\right| \leq\left(\sum_{j=j_{0}+1}^{\infty} c_{j}^{2}\right)^{1 / 2}\left(\sum_{j=j_{0}+1}^{\infty} r_{k, j}^{2}\right)^{1 / 2}<\varepsilon
$$

for all $k$, and $\sum_{j=j_{0}+1}^{\infty}\left|c_{j} r_{j}\right|<\varepsilon$. By Lemma 26, we can take sufficiently large $k_{0}$ such that, if $k \geq k_{0}$, then

$$
\left|\sum_{j=1}^{j_{0}} c_{j} r_{k, j}-\sum_{j=1}^{j_{0}} c_{j} r_{j}\right|<\varepsilon
$$

Thus, if $k \geq k_{0}$, then we have

$$
\begin{aligned}
\left|\sum_{j=1}^{\infty} r_{k, j} c_{j}-\sum_{j=1}^{\infty} r_{j} c_{j}\right| \leq & \left|\sum_{j=1}^{j_{0}} r_{k, j} c_{j}-\sum_{j=1}^{j_{0}} r_{j} c_{j}\right| \\
& +\left|\sum_{j=j_{0}+1}^{\infty} r_{k, j} c_{j}\right|+\left|\sum_{j=j_{0}+1}^{\infty} r_{j} c_{j}\right|<3 \varepsilon,
\end{aligned}
$$

which implies that $h\left(f\left(\mathbf{x}_{k}\right), f(\mathbf{x})\right) \longrightarrow 0$. This copmpletes the proof of the continuity of the functional $f$.

Take arbitrary elements $\mathbf{x}, \mathbf{y} \in \mathbb{V}$, and put $x_{j}=\mathbf{x} \cdot \mathbf{e}_{j}, y_{j}=$ $\mathbf{y} \cdot \mathbf{e}_{j}$ for $j=1,2, \cdots$. Then, it follows from the definition of $\oplus_{1}$ that

$$
\begin{aligned}
s\left(\frac{\mathbf{x}}{s} \oplus_{1} \frac{\mathbf{y}}{s}\right) \cdot \mathbf{e}_{j} \\
\quad=\frac{\left(1+\left(2 / s^{2}\right) \mathbf{x} \cdot \mathbf{y}+\left(1 / s^{2}\right)\|\mathbf{y}\|^{2}\right) x_{j}+\left(1-\left(1 / s^{2}\right)\|\mathbf{x}\|^{2}\right) y_{j}}{1+\left(2 / s^{2}\right) \mathbf{x} \cdot \mathbf{y}+\left(1 / s^{4}\right)\|\mathbf{x}\|^{2}\|\mathbf{y}\|^{2}} \\
\quad \longrightarrow x_{j}+y_{j}
\end{aligned}
$$


as $s \longrightarrow \infty$. For sufficiently large $s>0$, applying the orthogonal gyroexpansion, there exists a unique sequence $\left(r_{1}(s)\right.$, $\left.r_{2}(s), \cdots\right)$ of real numbers such that

$$
\frac{\mathbf{x}}{s} \oplus_{1} \frac{\mathbf{y}}{s}=\sum_{j=1}^{\infty} r_{j}(s) \otimes_{1} \frac{\mathbf{e}_{j}}{2}=\sum_{j=1}^{\infty} t_{j}(s) \mathbf{e}_{j}
$$

where we put $t_{j}(s)=\tanh \left(r_{j}(s) \tanh ^{-1}(1 / 2)\right)$. Then, we have

$$
\left\|\frac{\mathbf{x} \oplus_{s} \mathbf{y}}{s}\right\|^{2}=\left\|\frac{\mathbf{x}}{\mathcal{S}} \oplus_{1} \frac{\mathbf{y}}{s}\right\|^{2}=\left\|\sum_{j=1}^{\infty} t_{j}(s) \mathbf{e}_{j}\right\|^{2}=\sum_{j=1}^{\infty} t_{j}(s)^{2}
$$

It follows from $\mathbf{x} \oplus_{s} \mathbf{y} \longrightarrow \mathbf{x}+\mathbf{y}$ that $\left\|\mathbf{x} \oplus_{s} \mathbf{y} / s\right\| \longrightarrow 0$ and hence $t_{j}(s) \longrightarrow 0$ as $s \longrightarrow \infty$. Put $\mathbf{z}_{1}(s)=\sum_{j=2}^{\infty} \oplus_{1} t_{j}(s) \mathbf{e}_{j}$. Note that $\left\|\mathbf{z}_{1}(s)\right\| \leq\left\|\mathbf{x} \oplus_{s} \mathbf{y} / s\right\| \longrightarrow 0$ as $s \longrightarrow \infty$.

$$
\begin{aligned}
\left(\frac{\mathbf{x}}{s} \oplus_{1} \frac{\mathbf{y}}{s}\right) \cdot \mathbf{e}_{1} & =\left\{t_{1}(s) \mathbf{e}_{1} \oplus_{1} \mathbf{z}_{1}(s)\right\} \cdot \mathbf{e}_{1} \\
& =\frac{\left(1+\left\|\mathbf{z}_{1}(s)\right\|^{2}\right) t_{1}(s) \mathbf{e}_{1}+\left(1-t_{1}(s)^{2}\right) \mathbf{z}_{1}(s)}{1+t_{1}(s)^{2}\left\|\mathbf{z}_{1}(s)\right\|^{2}} \cdot \mathbf{e}_{1} \\
& =\frac{\left(1+\left\|\mathbf{z}_{1}(s)\right\|^{2}\right) t_{1}(s)}{1+t_{1}(s)^{2}\left\|\mathbf{z}_{1}(s)\right\|^{2}} \\
\left(\mathbf{x} \oplus_{s} \mathbf{y}\right) \cdot \mathbf{e}_{1} & =\frac{\left(1+\left\|\mathbf{z}_{1}(s)\right\|^{2}\right) s t_{1}(s)}{1+t_{1}(s)^{2}\left\|\mathbf{z}_{1}(s)\right\|^{2}}
\end{aligned}
$$

By letting $s \longrightarrow \infty$ in the formula above, we have $s t_{1}(s)$ $\longrightarrow(\mathbf{x}+\mathbf{y}) \cdot \boldsymbol{e}_{1}=x_{1}+y_{1}$.

Assume that we have shown $s t_{j}(s) \longrightarrow x_{j}+y_{j}(j=1, \cdots, n)$.

$$
\begin{aligned}
\{- & \left.\left(s t_{1}(s) \mathbf{e}_{1} \oplus_{s} \cdots \oplus_{s} s t_{n}(s) \mathbf{e}_{n}\right) \oplus_{s}\left(\mathbf{x} \oplus_{s} \mathbf{y}\right)\right\} \cdot \mathbf{e}_{n+1} \\
= & \frac{\left(1+\left\|\mathbf{z}_{n+1}(s)\right\|^{2}\right) s t_{n+1}(s)}{1+t_{n+1}(s)^{2}\left\|\mathbf{z}_{n+1}(s)\right\|^{2}}
\end{aligned}
$$

where we put $\mathbf{z}_{n+1}(s)=\sum_{j=n+2}^{\infty}{ }^{\oplus_{1}} t_{j}(s) \mathbf{e}_{j}$. From the assumption of induction and Lemma 19, it follows that

$$
\begin{aligned}
s t_{n+1}(s) & \longrightarrow\left\{-\left(\left(x_{1}+y_{1}\right) \mathbf{e}_{1}+\cdots+\left(x_{n}+y_{n}\right) \mathbf{e}_{n}\right)+(\mathbf{x}+\mathbf{y})\right\} \cdot \mathbf{e}_{n+1} \\
& =x_{n+1}+y_{n+1} .
\end{aligned}
$$

Now, it is straightforward to see

$$
\begin{aligned}
f_{s}\left(\mathbf{x} \oplus_{s} \mathbf{y}\right) & =s f\left(\frac{\mathbf{x} \oplus_{s} \mathbf{y}}{s}\right)=s f\left(\frac{\mathbf{x}}{s} \oplus_{1} \frac{\mathbf{y}}{s}\right) \\
& =s\left\{\left(\sum_{j=1}^{\infty} c_{j} r_{j}(s)\right) \otimes_{1} \frac{1}{2}\right\}=\left(\sum_{j=1}^{\infty} c_{j} r_{j}(s)\right) \otimes_{s} \frac{s}{2} \\
& =\sum_{j=1}^{\infty \otimes_{s}}\left(c_{j} r_{j}(s) \otimes_{s} \frac{s}{2}\right)=\sum_{j=1}^{\infty}\left(c_{j} \otimes_{s} s t_{j}(s)\right) .
\end{aligned}
$$




\section{References}

[1] A. A. Ungar, "Thomas rotation and the parametrization of the Lorentz transformation group," Foundations of Physics Letters, vol. 1, no. 1, pp. 57-89, 1988.

[2] A. A. Ungar, "Group-like structure underlying the unit ball in real inner product spaces," Results in Mathematics, vol. 18, no. 3-4, pp. 355-364, 1990.

[3] A. A. Ungar, "Extension of the unit disk gyrogroup into the unit ball of any real inner product space," Journal of Mathematical Analysis and Applications, vol. 202, no. 3, pp. 10401057, 1996.

[4] T. Abe and O. Hatori, "Generalized gyrovector spaces and a Mazur-Ulam theorem," Publicationes Mathematicae Debrecen, vol. 87, no. 3-4, pp. 393-413, 2015.

[5] O. Hatori, "Examples and applications of generalized gyrovector spaces," Results in Mathematics, vol. 71, no. 1-2, pp. 295317, 2017.

[6] T. Abe, "Normed gyrolinear spaces: a generalization of normed spaces based on gyrocommutative gyrogroups," Mathematics Interdisciplinary Research, vol. 1, no. 1, pp. 143-172, 2016.

[7] T. Abe and K. Watanabe, "Finitely generated gyrovector subspaces and orthogonal gyrodecomposition in the Möbius gyrovector space," Journal of Mathematical Analysis and Applications, vol. 449, no. 1, pp. 77-90, 2017.

[8] J. B. Conway, A Course in Functional Analysis, vol. 96 of Graduate Texts in Mathematics, Springer-Verlag, New York, 2nd edition, 1990.

[9] A. A. Ungar, Analytic Hyperbolic Geometry. Mathematical foundations and applications, World Scientific Publishing Co Pte Ltd, Hackensack, NJ, 2005.

[10] A. A. Ungar, Analytic Hyperbolic Geometry and Albert Einstein's Special Theory of Relativity, World Scientific Publishing Co Pte Ltd, Singapore, 2008.

[11] K. Watanabe, "Orthogonal Gyroexpansion in Möbius Gyrovector Spaces," Journal of Function Spaces, vol. 2017, Article ID 1518254, 13 pages, 2017.

[12] K. Watanabe, "A Cauchy type inequality for Möbius operations," Journal of Inequalities and Applications, vol. 2018, Article ID 97, 2018.

[13] K. Watanabe, "A Cauchy-Bunyakovsky-Schwarz type inequality related to the Möbius addition," Journal of Mathematical Inequalities, vol. 12, no. 4, pp. 989-996, 2018.

[14] K. Watanabe, "Cauchy-Bunyakovsky-Schwarz type inequalities related to Möbius operations," Journal of Inequalities and Applications, vol. 2019, Article ID 179, 2019. 